\title{
Increased Expression of Ganglioside GM1 in Peripheral CD4+ T Cells Correlates Soluble Form of CD30 in Systemic Lupus Erythematosus Patients
}

\author{
Lingli Dong, ${ }^{1}$ Shaoxian Hu, ${ }^{1}$ Fang Chen, ${ }^{1}$ Xiaomei Lei, ${ }^{1}$ Wei Tu, ${ }^{1}$ Yikai Yu, ${ }^{1}$ Liu Yang, \\ Wei Sun, ${ }^{2}$ Takuro Yamaguchi, ${ }^{3}$ Yasufumi Masaki, ${ }^{4}$ and Hisanori Umehara ${ }^{4}$ \\ ${ }^{1}$ Department of Rheumatology and Immunology, Tongji Hospital, Tongji Medical College, Huazhong University of \\ Science and Technology, 1095th Jiefang Avenue, Wuhan, Hubei 430030, China \\ ${ }^{2}$ Department of Stomatology, Union Hospital, Tongji Medical College, Huazhong University of Science and Technology, \\ 1277th Jiefang Avenue, Wuhan, Hubei 430022, China \\ ${ }^{3}$ Department of Clinical Laboratory, Kansai Electric Power Hospital, Osaka 555-0003, Japan \\ ${ }^{4}$ Department of Hematology and Immunology, Kanazawa Medical University, Ishikawa 920-0265, Japan
}

Correspondence should be addressed to Lingli Dong, dongll@tjh.tjmu.edu.cn

Received 3 February 2010; Revised 14 April 2010; Accepted 26 April 2010

Academic Editor: Brian Poole

Copyright ( 2010 Lingli Dong et al. This is an open access article distributed under the Creative Commons Attribution License, which permits unrestricted use, distribution, and reproduction in any medium, provided the original work is properly cited.

Gangliosides GM1 is a good marker of membrane microdomains (lipid rafts) with important function in cellular activation processes. In this study we found that GM1 expression on CD4+ T cells and memory T cells (CD45RO/CD4) were dramatic increased after stimulation with phytohaemagglutinin in vitro. Next, we examined the GM1 expression on peripheral blood CD4+ $\mathrm{T}$ cells and CD8+ T cells from 44 patients with SLE and 28 healthy controls by flow cytometry. GM1 expression was further analyzed with serum soluble CD30 (sCD30), IL-10, TNF-alpha and clinical parameters. The mean fluorescence intensity of GM1 on CD4+ T cells from patients with SLE was significantly higher than those from healthy controls, but not on CD8+ T cells. Increased expression of GM1 was more marked on CD4+/CD45RO+ memory T cells from active SLE patients. Patients with SLE showed significantly elevated serum sCD30 and IL-10, but not TNF-alpha levels. In addition, we found that enhanced GM1 expression on CD4+ T cells from patients with SLE positively correlated with high serum levels of sCD30 and IgG as well as disease activity (SLEDAI scores). Our data suggested the potential role of aberrant lipid raft/GM1 on CD4+ T cells and sCD30 in the pathogenesis of SLE.

\section{Introduction}

Systemic lupus erythematosus (SLE) is a multisystem, autoimmune, connective-tissue disorder in which organs and cells undergo damage mediated by tissue-binding autoantibodies and immune complexes [1]. T cells from SLE patients are known to provide help to $\mathrm{B}$ cells to produce autoantibodies and numerous abnormalities of $\mathrm{T}$ cells including aberrancies of proliferation, cell death, signaling, and cytokines production have been described in the pathogenesis of SLE $[2,3]$.

Lipid rafts are liquid ordered sphingolipid and cholesterol-enriched membrane domains, functioning in cellular processes, especially in signal transduction through recruiting signaling and stimulatory proteins [4], and play a critical role in $\mathrm{T}$ lymphocytes activation, particularly in signaling from the $\mathrm{T}$-cell antigen receptor (TCR) and in localization and function of proteins residing proximal to the receptor [4-7]. Ganglioside GM1, a major constituent of cellular membranes acting as a rigid barrier to the extracellular environment, is one of the important component of lipid raft [4]. Elevated GM1 have been observed in activated T-cells [8]. High levels of GM1 and cholesterol have been found in peripheral blood T cells from SLE patients, which was only measured in whole negatively selected $\mathrm{T}$ cells population by confocal microscopy study [9]. However, the levels of GM1 in T cell subgroups such as CD4+ helper T cells and CD8+ cytotoxic T cells are largely unknown. 
CD30, a 120-kDa type I transmembrane glycoprotein, is normally found on a subset of activated $\mathrm{T}$ cells, which are involved in the induction of cell proliferation and initiation of apoptosis [10]. The soluble form of CD30 ( $\mathrm{sCD} 30)$ is released from activated T cells through proteolytic cleavage. Elevated serum levels of sCD30 have been found in Hodgkin's disease, anaplastic large cell lymphoma, infectious and allergic diseases as well as some autoimmune diseases, such as SLE and rheumatoid arthritis $[11,12]$. However, its relationship with the membrane raft GM1 and cytokines in SLE has not been defined. To analyze lipid raft expression on each subgroup $\mathrm{T}$ cells in SLE and its relation to abnormal $\mathrm{T}$ cell activation and cytokine production, we quantified GM1 expression on both peripheral CD4+ and CD8+ T cells from the SLE patients via flow cytometry and compared it to the serum levels of sCD30 and Th1/Th2 balance of cytokines as well as clinical parameters. We found that GM1 expression was increased on CD4+ T cells and there were significant correlations between GM1 expression and sCD30 and disease activity in SLE.

\section{Materials and Methods}

2.1. Patients and Healthy Controls. Forty-four consecutive patients fulfilling the revised American College of Rheumatology (ACR) criteria for the diagnosis of SLE [13] were recruited for this study. Twenty-eight age-matched healthy volunteers served as controls. Disease activity was scored and the SLE Disease Activity Index (SLEDAI) was calculated based on previous report [14]. Patients were divided into subgroups according to clinical disease activity (i.e., active $\geq 10$ and inactive $<10$ by SLEDAI) and serum IgG level (high IgG $>15 \mathrm{~g} / \mathrm{L}$ ), respectively. Our study included 21 active SLE patients, 23 inactive patients with SLEDAI ranging from one to 20 , and 28 healthy control volunteers. Written informed consent was obtained from all participating patients and volunteers. Complete blood cell count, serum complement, serum IgG, antinuclear and anti-DNA antibodies were measured in all patients.

2.2. FACS Analysis. Surface expression of CD4, CD8, CD45RO, and GM1 were analyzed from the peripheral blood cells by double or triple-staining using FITC-, PE-, APC-labeled mAbs in the relevant combinations. Two color immunofluorescence analysis was performed on a FACScan flow cytometer (BD Biosciences, San Jose, CA), using CellQuest Pro (BD Biosciences) software. Detection of GM1 expression was performed by staining peripheral blood with PE-conjugated anti-CD4 or CD8 antibodies (eBioscience Co. Ltd, USA) and FITC-conjugated cholera toxin-B (CTB, Sigma-Aldrich, USA) as described previously [15]. For triple staining, cells were first analyzed by APC-conjugated CD4, then GM1 expression was detected with PE anti-CD45RO antibody and FITC cholera toxin-B.

2.3. Expression of GM1 on PHA-Activated T Cells during Culture. Mononuclear cells were isolated from heparinized peripheral blood from the healthy subjects by densitygradient centrifugation on Ficoll-Hypaque (Pharmacia, Uppsala, Sweden). The cells were then stimulated with $1 \mu \mathrm{g} / \mathrm{ml}$ of PHA (Boehringer Mannheim, Germany) and maintained in RPMI 1640 medium supplemented with 10\% FCS (Upstate Biotechnology, Inc., UBI, Lake Placid, $\mathrm{NY}$ ), $2 \mathrm{mM}$ L-glutamine, penicillin, and streptomycin in the presence of $2 \mathrm{nM}$ recombinant IL-2 (Shionogi Co. Ltd., Osaka, Japan) for 2 weeks. To analyze surface expression of ganglioside GM1 and CD45RO on CD4+ T cells, cells were stained with FITC-conjugated CTB (Sigma-Aldrich), PEconjugated anti-CD45RO and APC-conjugated anti-CD4 mAbs (BD Bioscience) on ice for 30 minutes, respectively, and analyzed with a FACSan. Data were analyzed using CellQuest software (Becton Dickinson).

2.4. ELISA. The serum levels of sCD30, IL-10, and TNF$\alpha$ were measured by an enzyme-linked immunosorbent assay (ELISA) commercial kit (R\&D Co. Ltd. USA) [16, 17]. The serum IgG of the SLE patients was detected by immunoturbidimetric assay (Orion Diagnostica Oy Co. Ltd. Finland). The results were subjected to the independent sample $t$-test, correlation tests, and bivariate analysis. A value of $P<.05$ was considered to be statistically significant.

\section{Results}

3.1. GM1 Expressions on CD4+ T Cells and Memory T Cells (CD45RO/CD4) Were Dramaticly Increased after Stimulation with PHA In Vitro. CTB binds the raft-associated GM1, previously shown to be a reliable marker for detection of lipid raft domains [18]. First, we analyzed the rafts on mitogenstimulated $\mathrm{T}$ cells in vitro through FACS. GM1 surface expression with fluorescent-labeled CTB was determined on days $0,2,5,8,11$ and 14 after stimulation with $1 \mu \mathrm{g} / \mathrm{ml}$ of PHA. The mean fluorescence intensity (MFI) of memory $\mathrm{T}$ cells (CD45RO/CD4) were dramatically increased in timedependent manner after stimulation (Figures 1(a) and 1(b)) as well as CD4+ T cells (Figure 1(c)). These data indicate that cellular membrane adopts an increased lipid raft structure upon mitogenic stimulation in CD4+ $\mathrm{T}$ cells and CD4+ memory T cells.

3.2. Elevated Expression of GM1 Was Presented on CD4+ T Cells, Not on CD8+ T Cells from SLE Patient Peripheral Blood. Next, we examined the expression of GM1 on peripheral blood CD4+ T cells and CD8+ T cells from 44 patients with SLE and 28 healthy controls by flow cytometry. The MFI of GM1 on peripheral CD4+ T cells from patients with SLE were significantly stronger than those from the healthy controls $(P<.01)$ (Figure $2(\mathrm{a}))$. Although the increase of CD4+ T cells expressing GM1 was small, the substantial change in GM1 expression as measured by MFI suggests that increased GM1 expression affects a high proportion of lupus CD4+ T cells. When we divided SLE patients into active group $(n=21)$ and inactive group $(n=23)$ according to SLEDAI scores (active $\geq 10$, inactive $<10$ ), MFI of GM1 in both groups are higher than those of healthy 


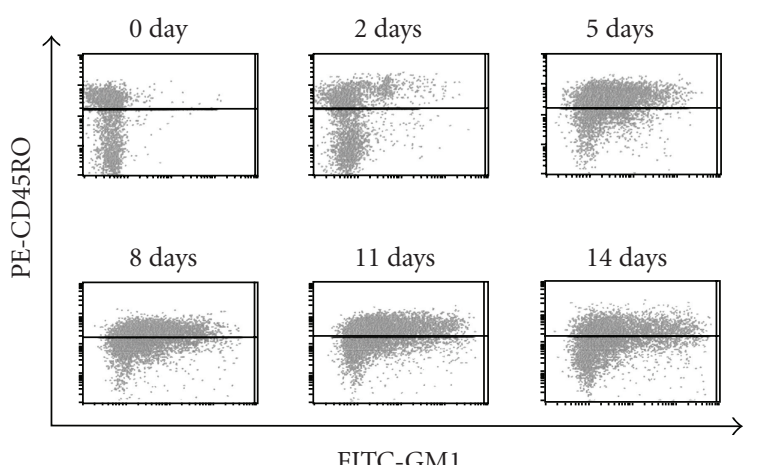

(a)

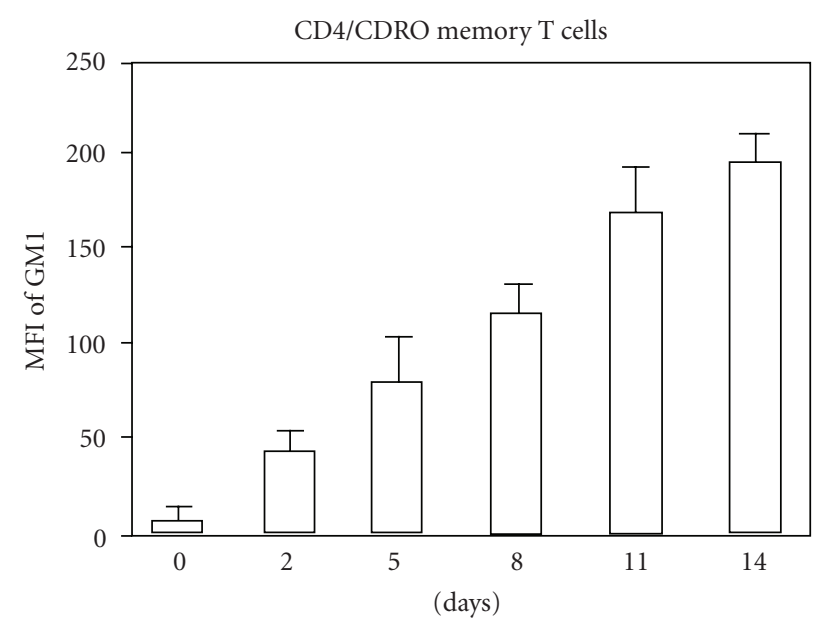

(b)

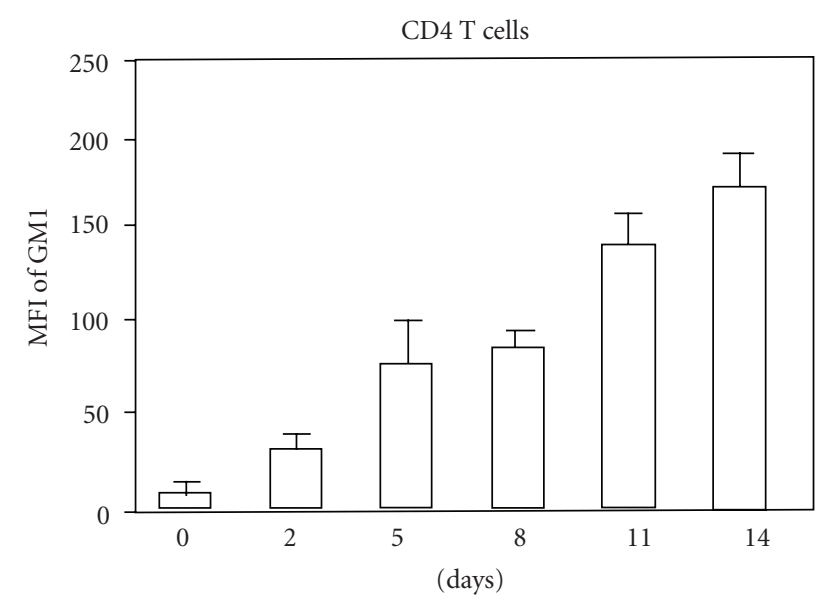

(c)

FIgure 1: Time kinetics of GM1 expressions on PHA-activated T cells. Peripheral mononuclear cells were stimulated with $1 \mu \mathrm{g} / \mathrm{ml}$ of PHA and maintained with IL-2-containing medium for two weeks. GM1 expression on memory T cells were detected with FITC-CTB and PECD45RO antibody on days $0,2,5,8,11$ and 14 after stimulation (a). The mean fluorescence intensity (MFI) of GM1 were measured on CD45RO+/CD4+ memory T cells (b) and CD4+ T cells (c). Each experiment was done in triplicate. Error bars represent S.E.M.

controls. Although the difference was not significant, MFI of GM1 in active group is higher than that in inactive group (Figure 2(a)). When we divided SLE patients into high IgG group $(n=24)$ and normal IgG group $(n=20)$ according to serum IgG level (>15 g/L), MFI of GM1 on CD4+ T cells from high IgG group was statistically higher than that from normal IgG group $(P<.05)$ (Figure $2(\mathrm{a}))$. In contrast to $\mathrm{CD} 4+\mathrm{T}$ cells, no significant difference in expression of GM1 on CD8+ T cells was observed between SLE patients and the healthy controls (Figure 2(b)).

Further, we analyzed GM1 expression on $\mathrm{CD} 45 \mathrm{RO}+/ \mathrm{CD} 4+$ memory $\mathrm{T}$ cells from 11 of inactive SLE, 14 of active SLE patients and 23 healthy controls by triple staining (Figures 2(c)-2(e)). Figure 2(c) shows that there are no significant differences in percentage of CD4 $\mathrm{T}$ cells among inactive SLE $(42.2 \pm 3.04)$, active SLE $(38.8 \pm 2.64)$, and healthy controls $(46.9 \pm 2.17)$. We did not found the significant difference of the MFI of GM1 on $\mathrm{CD} 45 \mathrm{RO}+/ \mathrm{CD} 4+$ memory $\mathrm{T}$ cells among each group (data not shown). However, when we determined the cell with more than 10 in MFI of GM1 as high GM1 cells (Figure 2(d)), percent of high GM1 cells among $\mathrm{CD} 45 \mathrm{RO}+\mathrm{CD} 4+$ memory T cells was significantly increased in SLE patients $(1.00 \pm 0.07$ and $1.35 \pm 0.12$ for inactive and active SLE, resp.) compared with healthy controls $(0.69 \pm 0.03)$ (Figure $2(\mathrm{e}))$. Although the change in the percentage of high GM1 cells was small, this represented a twofold increase compared with those from healthy individuals. Moreover, percent of high GM1 cells in active SLE is significantly higher than that in inactive SLE (Figure 2(e)). The elevation in high GM1 cells of memory $\mathrm{T}$ cells was dependent of lupus disease activity status.

3.3. The Serum Levels of $s C D 30$ and IL-10 Were Significantly Increased in the SLE Patients, but TNF- $\alpha$ Was Not. The serum levels of sCD30 were averaged $149.74 \pm 63.85 \mathrm{ng} / \mathrm{ml}$ in the SLE patients $(149.25 \pm 66.83 \mathrm{ng} / \mathrm{ml}$ in active patients, 


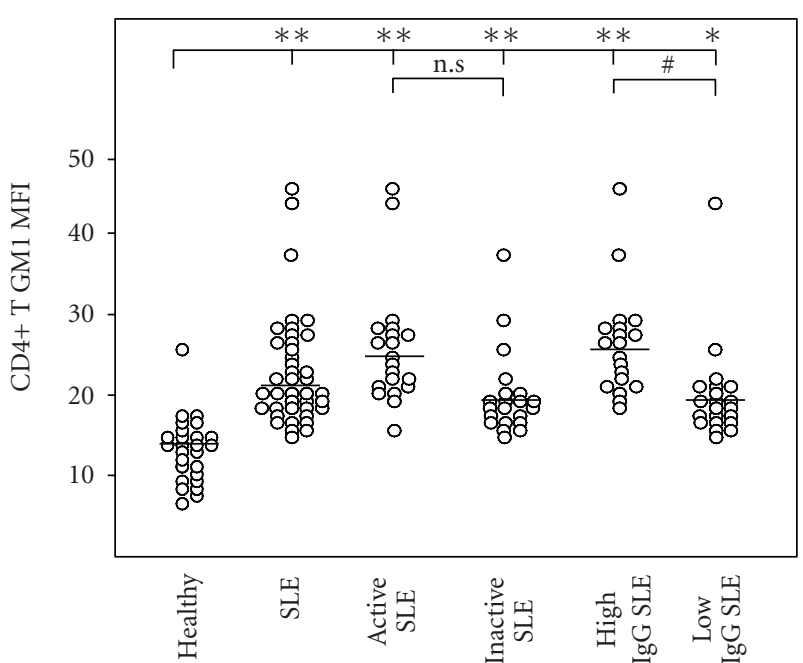

(a)

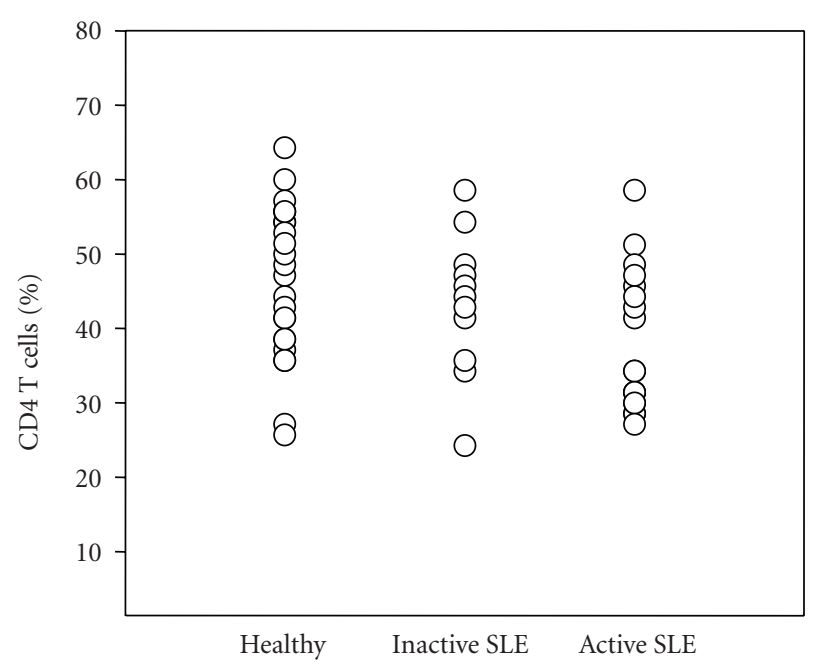

(c)
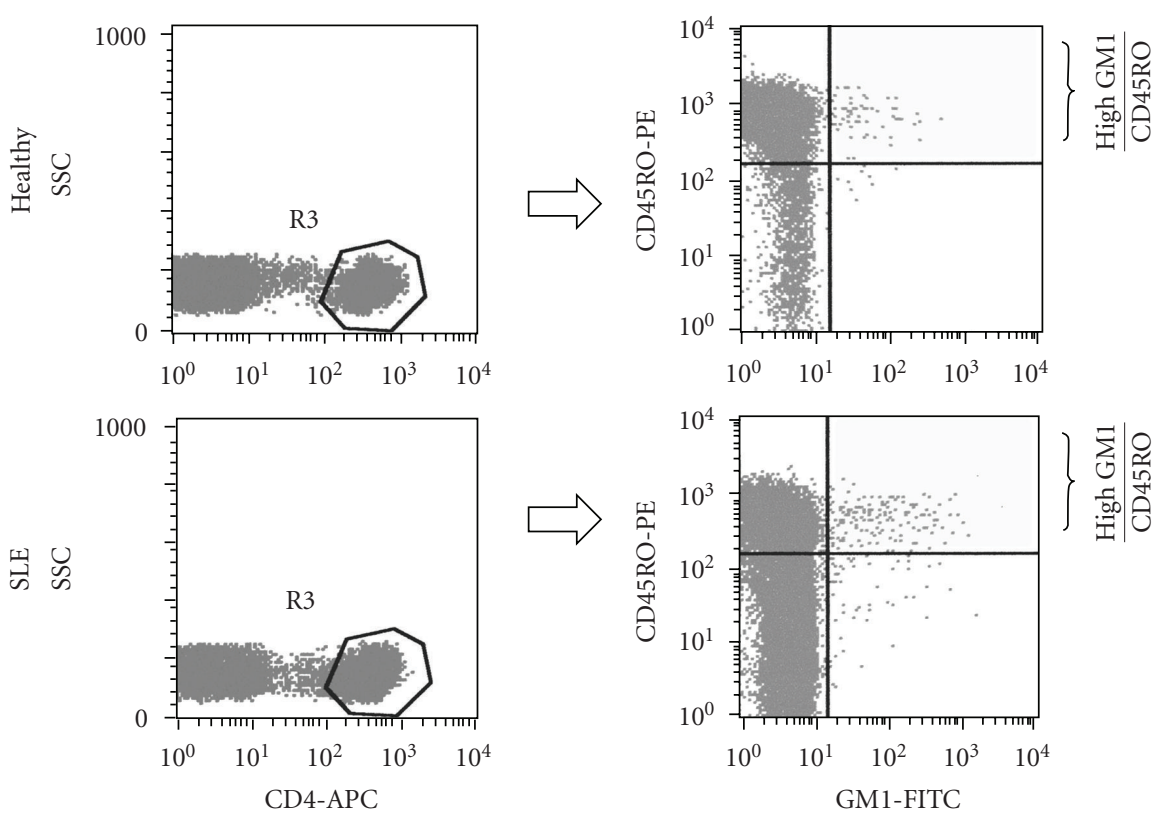

(d)

FIgure 2: Continued.

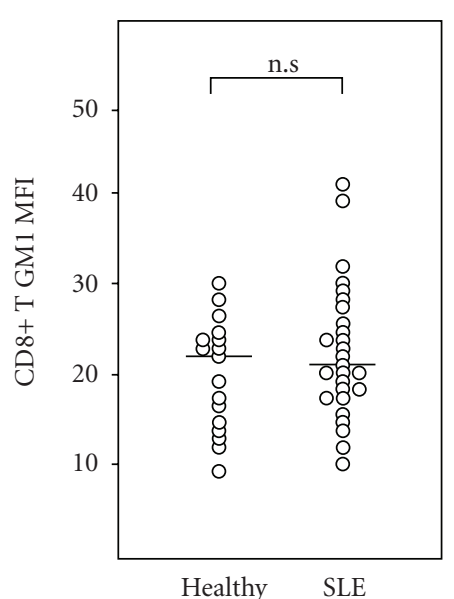

(b) 


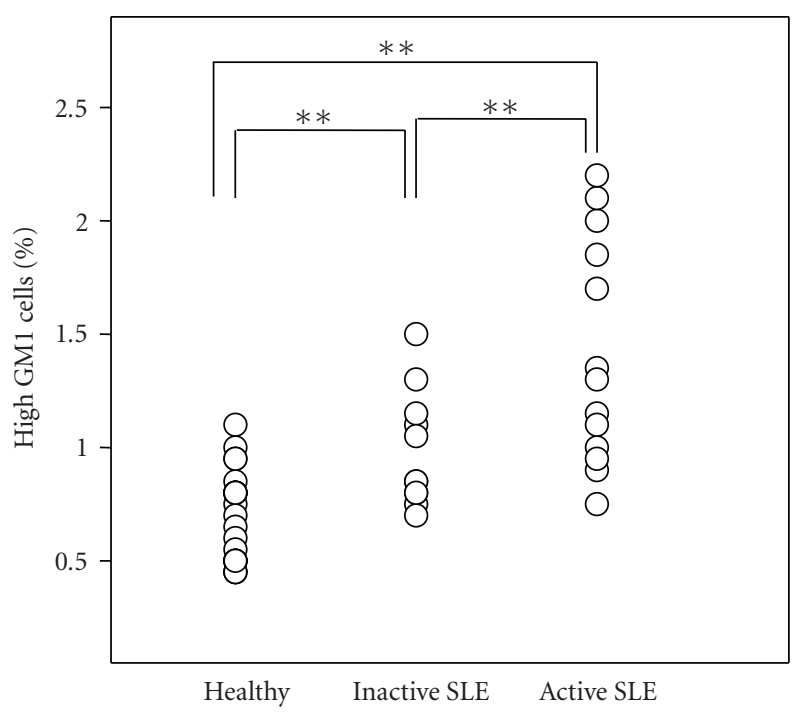

(e)

FIgure 2: Lipid raft/GM1 expression on CD4+ T cells (a) and CD8+ T cells (b). SLE patients were divided into subgroups; active SLE group $(n=21)$ versus inactive SLE group $(n=23)$ according to SLEDAI scores, and high IgG group $(n=24)$ versus normal IgG group $(n=20)$ according to serum IgG level. ${ }^{*}$ Statistically significant differences compared to the healthy $\left({ }^{*} P<.05,{ }^{* *} P<.01\right)$. \#Statistically significant differences between subgroup with high IgG and normal IgG. $(P<.01)$ (Two-tailed unpaired Student's $t$-test). (c) Percentage of CD4-positive T cells among healthy controls $(n=23)$, inactive $(n=11)$ and active SLE patients $(n=13)$ were $46.9+2.17,42.2+3.04$ and 38.8+2.64, respectively. (d) GM1 expression on CD45RO+/CD4+ memory T cells by triple staining. Cells were first gated by CD4-APC, then analyzed with PE-CD45RO antibody and FITC-GM1. Cells with more than 10 in MFI of GM1 were determined as high GM1 cells. (e) Percent of high GM1-expessing CD45RO+/CD4+ memory T cells among normal controls, inactive and active SLE patients $(0.69 \pm 0.03$, $1.00 \pm 0.07,1.35 \pm 0.12$, resp.). ${ }^{* *} P<.01$

$150.3 \pm 62.65 \mathrm{ng} / \mathrm{ml}$ in inactive patients), and were averaged $79.49 \pm 22.93 \mathrm{ng} / \mathrm{ml}$ in the healthy controls, showing a significant increase of serum sCD30 in the SLE patients, compared to healthy controls $(P<.01)$, although there was not difference between SLE active and inactive groups (Figure 3(a)). Levels of IL-10 were $94.6 \pm 57.93 \mathrm{pg} / \mathrm{ml}$ in the SLE patients $(89.07 \pm 46.44 \mathrm{pg} / \mathrm{ml}$ in active patients, $100.86 \pm$ $69.9 \mathrm{pg} / \mathrm{ml}$ in inactive patients) and $44.96 \pm 33.30 \mathrm{pg} / \mathrm{ml}$ in the healthy controls. There were statistical differences between the SLE patients and healthy controls $(P<.05)$ (Figure 3(b)). TNF- $\alpha$ were $18.62 \pm 9.57 \mathrm{pg} / \mathrm{ml}$ in the SLE patients $(19.35 \pm$ $9.15 \mathrm{pg} / \mathrm{ml}$ in the active patients, $17.80 \pm 10.20 \mathrm{pg} / \mathrm{ml}$ in the inactive patients) and $16.91 \pm 13.18 \mathrm{pg} / \mathrm{ml}$ in the healthy controls. There were no statistical differences in the levels of TNF- $\alpha$ between the SLE patients and the healthy controls (Figure 3(c)).

3.4. The Correlation among GM1 Expression on CD4+ T Cells, SLEDAI Scores, sCD30, IgG Levels and Other Clinic Parameters in the SLE Patients. Analyzing the correlations among the SLE patients, we found that there was a significant correlation between GM1 expression on CD4+ T cells (MFI), SLEDAI scores $(r=0.556, P<.005)$, plasma level of SCD30 $(r=0.51, P<.005)$, and serum IgG levels $(r=0.56$, $P<.005)$ in the SLE patients. In particular, the correlation between MFI of GM1 on CD4+ T cells and SLEDAI was much stronger in active SLE group $(r=0.813, P<.001)$ than those in inactive group $(r=0.668, P<.005)$. No
TABle 1: Correlations among the levels of sCD30 IgG, GM1 expression, and SLEDAI scores $\left({ }^{*} P<.005\right)$.

\begin{tabular}{lcccc}
\hline & GM1 & SLEDAI & sCD30 & IgG \\
\hline GM1 & 1 & & & \\
SLEDAI & $0.56^{*}$ & 1 & & \\
SCD30 & $0.51^{*}$ & NS & 1 & \\
IgG & $0.52^{*}$ & NS & $0.56^{*}$ & 1 \\
\hline
\end{tabular}

significant correlation was found between GM1 and IL-10, ANA titer, C3, and other clinic parameters (data not shown). Furthermore, we found that the plasma level of SCD30 was positively correlated with the IgG level (Table 1). But no statistical difference was observed between sCD30 levels and other parameters (data not shown). In addition, no statistical difference was observed between expression of GM1 on CD45RO+/CD4+ memory $\mathrm{T}$ cells and above parameters in patients with SLE.

\section{Discussion}

T-cell abnormalities and aberrant $\mathrm{T}$ helper cytokine profiles have been implicated in pathogenic process of SLE. Previous studies have demonstrated that negative selected peripheral blood T cells from the SLE patients have higher levels of GM1 [9]. In this study, we quantitatively measured membrane 


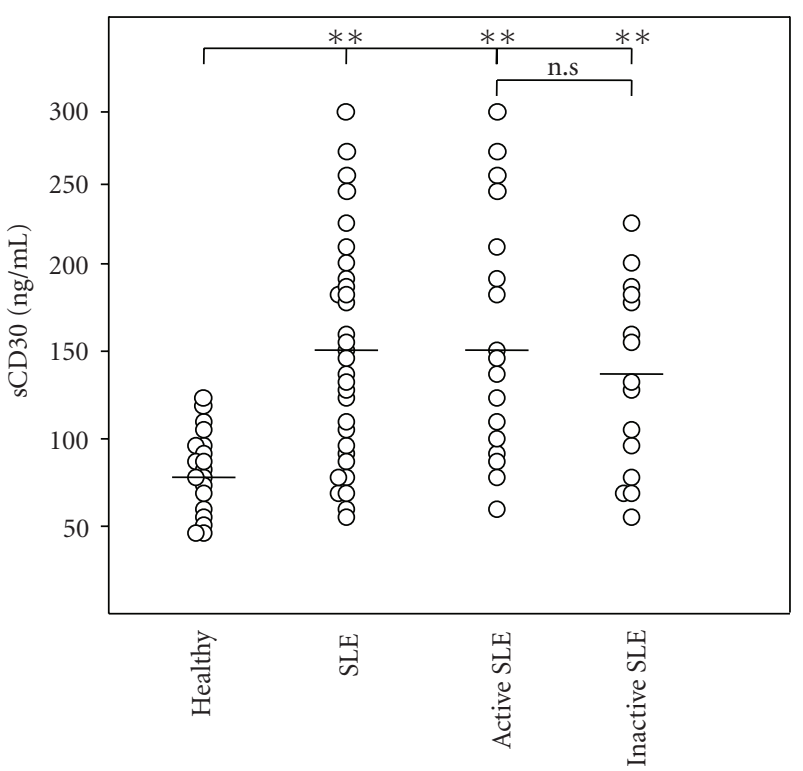

(a)

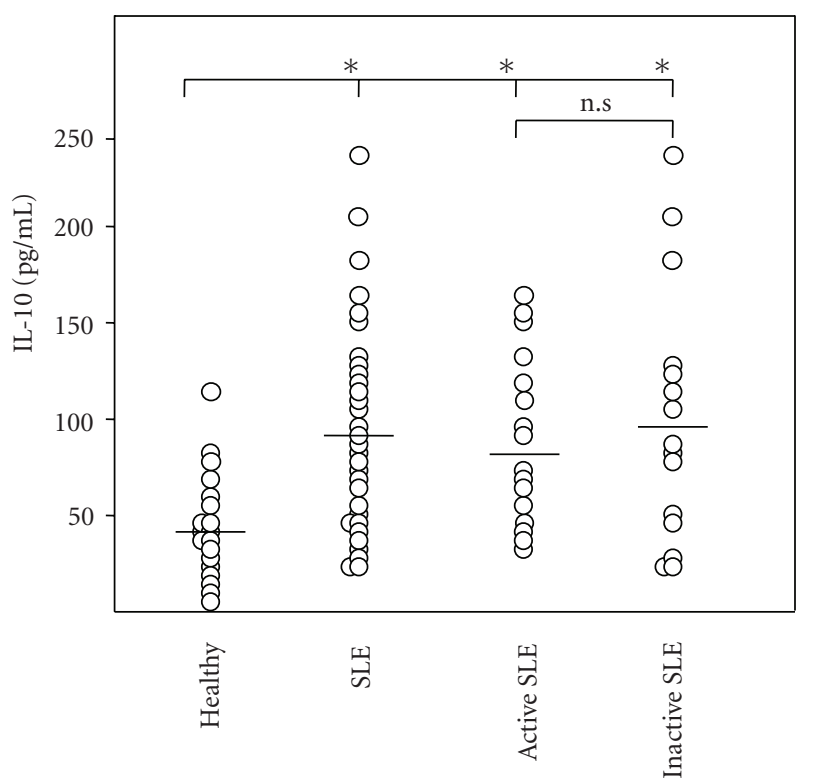

(b)

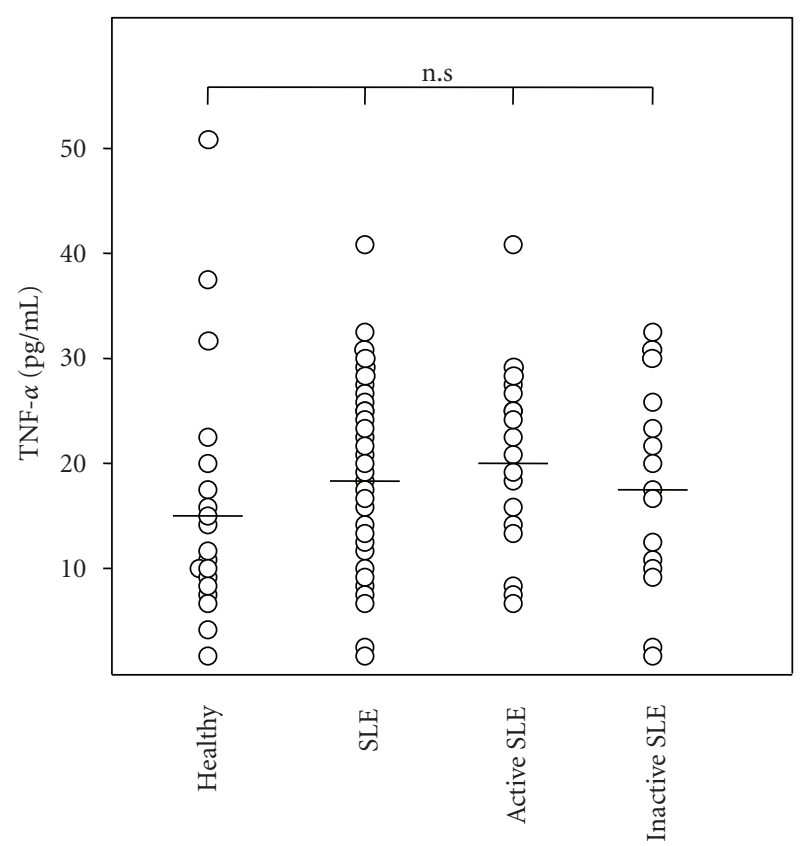

(c)

FIgURE 3: Serum levels of sCD30 (a), IL-10 (b) and TNF- $\alpha$ (c) in the SLE group, in the healthy controls, in the subgroups with active and inactive SLE. * Statistically significant differences between the healthy controls and the marked SLE group \& subgroups $(P<.01)$.

GM1 on peripheral blood CD4+ and CD8+ T cells, respectively, and found that CD4+ T cells expressed elevated GM1, but CD8+ T cells did not, in SLE patients. In addition, GM1 expressions on memory $\mathrm{T}$ cells $(\mathrm{CD} 45 \mathrm{RO} / \mathrm{CD} 4)$ and $\mathrm{CD} 4+$ $\mathrm{T}$ cells were dramaticly increased after PHA stimulation in vitro. Furthermore, number of GM1-highly expressing $\mathrm{CD} 45 \mathrm{RO}+\mathrm{CD} 4+$ memory $\mathrm{T}$ cells were significantly increased in SLE patients compared with healthy controls (Figure 2(e)). These data may indicate that cellular membrane adopts an increased lipid raft structure upon mitogenic stimulation and CD4+ T cells in SLE patients are continuously activated by autoantigens in vivo. It has been reported that CD8+ T cells in SLE are impaired and CD8+ T cells do not require the polarization of lipid rafts for activation and proliferation [19]. However, activated CD4+ helper T cells produce more cytokines and help B cells secrete autoantibodies that form immune complexes, resulting in subsequent inflammation and organ damage [20]. Additionally, we found that there was a positive correlation between GM1 expression on CD4+ $\mathrm{T}$ cells $(\mathrm{MFI})$ and SLEDAI scores $(r=0.556, P<.005)$ 
as well as serum IgG levels $(r=0.56, P<.005)$ in SLE group, suggesting a possible role of lipid raft GM1 in pathogenesis of SLE. It has been documented that SLE T cells possess dysregulated activity of multiple protein tyrosine kinases $[9,20,21]$. We hypothesize that high level of GM1 on $\mathrm{CD} 4+\mathrm{T}$ cells may induce changes in membrane density and segmentation impacting on signal strength. Previously, we reported that lipid rafts function as scaffolds for TCR signals by recruiting TCR, ZAP-70 and PKC- $\theta$ into rafts using membrane sphingomyelin knock down Jurkat cells [9]. These modulations may also facilitate that signaling, and the stimulatory proteins are recruited to or modified in lipid rafts, subsequently convey aberrant intracellular signals, resulting in abnormal $\mathrm{CD} 4+\mathrm{T}$ cell function [5]. These aberrances lead to the survival of autoimmune B clones and overproduction of antibodies, eventually contribute to the development of SLE [22].

Although CD30 originally described as a marker on Reed-Sternberg cells in Hodgkin lymphoma [10], CD30 can be found on various populations of $\mathrm{T}$ cells (both $\mathrm{CD} 4+$ and $\mathrm{CD} 8+$ cells), especially helper $\mathrm{T}$ cells which produce cytokines. The extracellular portion of CD30 is proteolytically cleaved from $\mathrm{CD} 30+$ cells, possibly on activation by $\mathrm{CD} 30 \mathrm{~L}$, to produce a soluble form of the molecule ( $\mathrm{sCD} 30$ ) detectable in serum [23]. The sCD30 belongs to the TNF superfamily and is important in the interaction and the activation of $\mathrm{T}$ and $\mathrm{B}$ cells. High levels of $\mathrm{sCD} 30$ have been seen in the cases of $\mathrm{T}$ helper immunodeficiency diseases, allergic diseases, the severe infections and autoimmune disease [23], and sCD30 has been proposed as a marker for a Th2-oriented immune response [12, 24]. Ciferská et al. reported that there was a significant differences of sCD30 at serum levels between the SLE patients and the controls [11]. We also found significant differences of serum sCD30 level between the SLE patients and healthy controls. Moreover, we found that there was a positive correlation between GM1 expression on CD4+ T cells (MFI) and serum level of SCD30 $(\mathrm{r}=0.51, P<.005)$. A Triton $\mathrm{X}-100$-based method for lipid raft isolation revealed that $\mathrm{CD} 30$ was partially present in lipid rafts [25]. Additionally, Bastian von Tresckow et al. found that cellular lipid raft such as cholesterol level regulate CD30 shedding in human Hodgkin lymphoma- and non-Hodgkin lymphoma derived cell line [25], implying the potential relationship between lipid raft and sCD30. Recently, sCD30 has been shown to be important for the regulation of the immune system in vivo and in vitro, and it is likely to play a regulatory role in the Th1/Th2 type immune response $[26,27]$. Increased serum levels of sCD30 in autoimmune disease demonstrated that the aberrant lipid raft is connected to an increased secretion of sCD30.

We further measured the TNF- $\alpha$ and IL-10 which respectively indicate Th1 and Th2 activation $[2,3,26]$. We found that the levels of IL-10 were significantly higher in SLE than those in healthy controls, however no significant difference of TNF- $\alpha$ was observed. Although the role of IL10 in SLE pathogenesis has not been completely elucidated, IL-10 is considered a regulatory Th2 cytokine, and it causes activation, differentiation, and antibody production in Bcells [3]. In addition, current studies reveal the existence of a subset of B cells with regulatory capacities. This regulatory subset is characterized by the secretion of IL-10 and transforming growth factor- $\beta$ (TGF- $\beta$ ) and has been shown to be involved in the pathogenesis of autoimmune diseases [28]. However, we did not found the positive correlation between IL-10 and sCD30, as well as other parameters. Our data suggest that SLE IL-10 is complex and has pleiotropic effects in immunoregulation and inflammation.

Taken together, we have found an aberrant GM1 expression on CD4+ T cells and significant correlations between GM1 expression and sCD30 and disease activity in SLE, indicating that lipid raft may become a direct target for modulation of the immune system. Additional research is needed to better understand the biologic basis of this effect.

\section{Acknowledgments}

This paper was supported by Grants from National Natutal Science Foundation of China (no. 30840006 and 30972706 to Dong), Tongji Hospital Research Foundation (to Dong), Uehara Memorial Foundation (to Umehara and Dong), The Vehicle Racing Commemorative Foundation (to Umehara), and Kanazawa Medical University Research Foundation (C2009-4 to Umehara).

\section{References}

[1] J. Yazdany, P. Panopalis, J. Z. Gillis, et al., "A quality indicator set for systemic lupus erythematosus," Arthritis \& Rheumatism, vol. 61, no. 3, pp. 370-377, 2009.

[2] R. W. Hoffman, "T cells in the pathogenesis of systemic lupus erythematosus," Clinical Immunology, vol. 113, no. 1, pp. 4-13, 2004.

[3] A. La Cava, "Lupus and T cells," Lupus, vol. 18, no. 3, pp. 196201, 2009.

[4] M. A. Alonso and J. Millán, "The role of lipid rafts in signalling and membrane trafficking in T lymphocytes," Journal of Cell Science, vol. 114, no. 22, pp. 3957-3965, 2001.

[5] P. S. Kabouridis and E. C. Jury, "Lipid rafts and T-lymphocyte function: implications for autoimmunity," FEBS Letters, vol. 582, no. 27, pp. 3711-3718, 2008.

[6] K. Jacobson, O. G. Mouritsen, and R. G. W. Anderson, "Lipid rafts: at a crossroad between cell biology and physics," Nature Cell Biology, vol. 9, no. 1, pp. 7-14, 2007.

[7] K. D. Mossman, G. Campi, J. T. Groves, and M. L. Dustin, "Altered TCR signaling from geometrically repatterned immunological synapses," Science, vol. 310, no. 5751, pp. 1191-1193, 2005.

[8] E. J. Pavón, P. Muñoz, M.-D.-C. Navarro, et al., "Increased association of CD38 with lipid rafts in T cells from patients with systemic lupus erythematosus and in activated normal $\mathrm{T}$ cells," Molecular Immunology, vol. 43, no. 7, pp. 1029-1039, 2006.

[9] E. C. Jury, P. S. Kabouridis, F. Flores-Borja, R. A. Mageed, and D. A. Isenberg, "Altered lipid raft-associated signaling and ganglioside expression in T lymphocytes from patients with systemic lupus erythematosus," Journal of Clinical Investigation, vol. 113, no. 8, pp. 1176-1187, 2004.

[10] M. P. Purdue, Q. Lan, O. Martinez-Maza, et al., "A prospective study of serum soluble CD30 concentration and risk of 
non-Hodgkin lymphoma," Blood, vol. 114, no. 13, pp. 2730 2732, 2009.

[11] H. Ciferská, P. Horák, Z. Heřmanová, et al., "The levels of sCD30 and of sCD40L in a group of patients with systemic lupus erythematodes and their diagnostic value," Clinical Rheumatology, vol. 26, no. 5, pp. 723-728, 2007.

[12] E. Savolainen, I. Matinlauri, H. Kautiainen, R. Luosujärvi, and O. Kaipiainen-Seppänen, "Serum soluble CD30 in early arthritis: a sign of inflammation but not a predictor of outcome," Clinical and Experimental Rheumatology, vol. 26, no. 5, pp. 922-925, 2008.

[13] E. M. Tan, A. S. Cohen, J. F. Fries, et al., "The 1982 revised criteria for the classification of systemic lupus erythrematosus," Arthritis and Rheumatism, vol. 25, no. 11, pp. 1271-1277, 1982.

[14] C. Bombardier, D. D. Gladman, M. B. Urowitz, D. Caron, and C. H. Chang, "Derivation of the SLEDAI. A disease activity index for lupus patients. The Committee on Prognosis Studies in SLE," Arthritis \& Rheumatism, vol. 35, pp. 630-640, 1992.

[15] Z.-X. Jin, C.-R. Huang, L. Dong, et al., "Impaired TCR signaling through dysfunction of lipid rafts in sphingomyelin synthase 1 (SMS1)-knockdown T cells," International Immunology, vol. 20, no. 11, pp. 1427-1437, 2008.

[16] L. Dong, Y. Masaki, T. Takegami, et al., "Clonality analysis of lymphoproliferative disorders in patients with Sjögren's syndrome," Clinical and Experimental Immunology, vol. 150, no. 2, pp. 279-284, 2007.

[17] L. Dong, Y. Masaki, T. Takegami, et al., "Cloning and expression of two human recombinant monoclonal Fab fragments specific for EBV viral capsid antigen," International Immunology, vol. 19, no. 3, pp. 331-336, 2007.

[18] P. W. Janes, S. C. Ley, and A. I. Magee, "Aggregation of lipid rafts accompanies signaling via the $\mathrm{T}$ cell antigen receptor," Journal of Cell Biology, vol. 147, no. 2, pp. 447-461, 1999.

[19] B. Kovacs, M. V. Maus, J. L. Riley, et al., "Human CD8+ T cells do not require the polarization of lipid rafts for activation and proliferation," Proceedings of the National Academy of Sciences of the United States of America, vol. 99, no. 23, pp. 1500615011, 2002.

[20] K. Tenbrock, Y.-T. Juang, V. C. Kyttaris, and G. C. Tsokos, "Altered signal transduction in SLE T cells," Rheumatology, vol. 46, no. 10, pp. 1525-1530, 2007.

[21] N. Noraz, K. Schwarz, M. Steinberg, et al., "Alternative antigen receptor (TCR) signaling in T cells derived from ZAP-70deficient patients expressing high levels of Syk," Journal of Biological Chemistry, vol. 275, no. 21, pp. 15832-15838, 2000.

[22] E. C. Jury, F. Flores-Borja, and P. S. Kabouridis, "Lipid rafts in T cell signalling and disease," Seminars in Cell and Developmental Biology, vol. 18, no. 5, pp. 608-615, 2007.

[23] N. Rezaei, M. Haji-Molla-Hoseini, A. Aghamohammadi, A. A. Pourfathollah, M. Moghtadaie, and Z. Pourpak, "Increased serum levels of soluble CD30 in patients with common variable immunodeficiency and its clinical implications," Journal of Clinical Immunology, vol. 28, no. 1, pp. 78-84, 2008.

[24] F. Caligaris-Cappio, M. T. Bertero, M. Converso, et al., "Circulating levels of soluble CD30, a marker of cells producing Th2type cytokines, are increased in patients with systemic lupus erythematosus and correlate with disease activity," Clinical and Experimental Rheumatology, vol. 13, no. 3, pp. 339-343, 1995.

[25] B. Von Tresckow, K.-J. Kallen, E. P. Von Strandmann, et al., "Depletion of cellular cholesterol and lipid rafts increases shedding of CD30," Journal of Immunology, vol. 172, no. 7, pp. 4324-4331, 2004.
[26] G. Solgi, A. A. Amirzagar, G. Pourmand, et al., "TH1/TH2 cytokines and soluble CD30 levels in kidney allograft patients with donor bone marrow cell infusion," Transplantation Proceedings, vol. 41, no. 7, pp. 2800-2804, 2009.

[27] R. Gerli, C. Lunardi, E. B. Bocci, et al., "Anti-tumor necrosis factor- $\alpha$ response in rheumatoid arthritis is associated with an increase in serum soluble CD30," Journal of Rheumatology, vol. 35, no. 1, pp. 14-19, 2008.

[28] S. Dolff, W. H. Abdulahad, M. Bijl, and C. G. M. Kallenberg, "Regulators of B-cell activity in SLE: a better target for treatment than B-cell depletion?" Lupus, vol. 18, no. 7, pp. 575-580, 2009. 


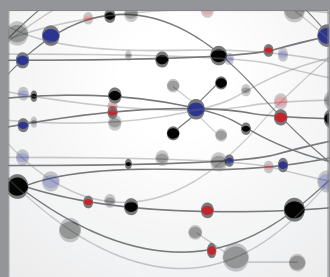

The Scientific World Journal
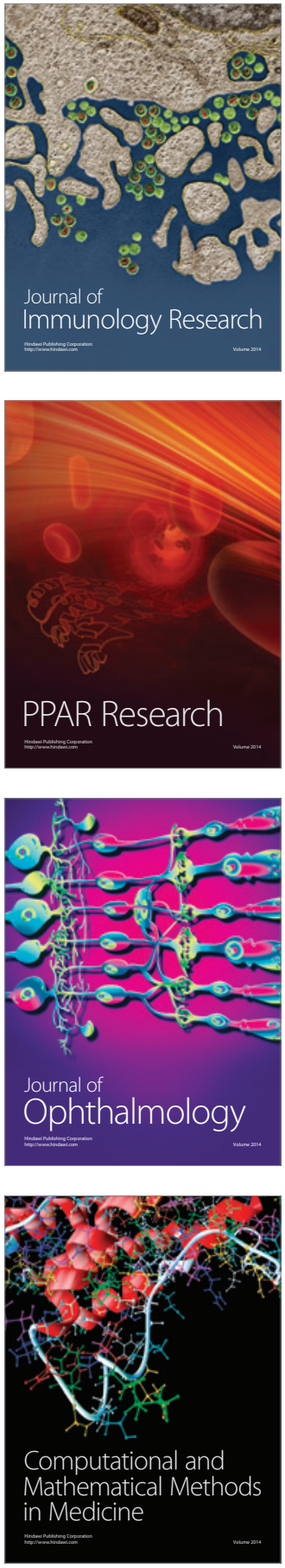

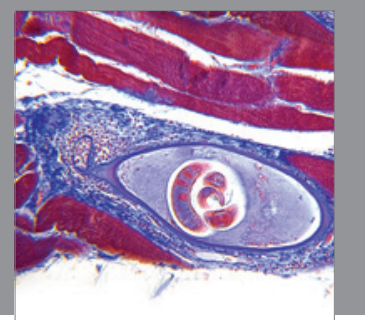

Gastroenterology

Research and Practice
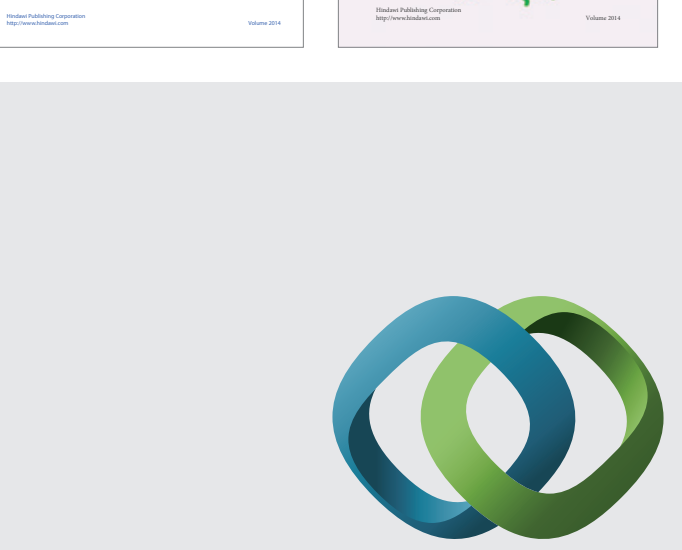

\section{Hindawi}

Submit your manuscripts at

http://www.hindawi.com
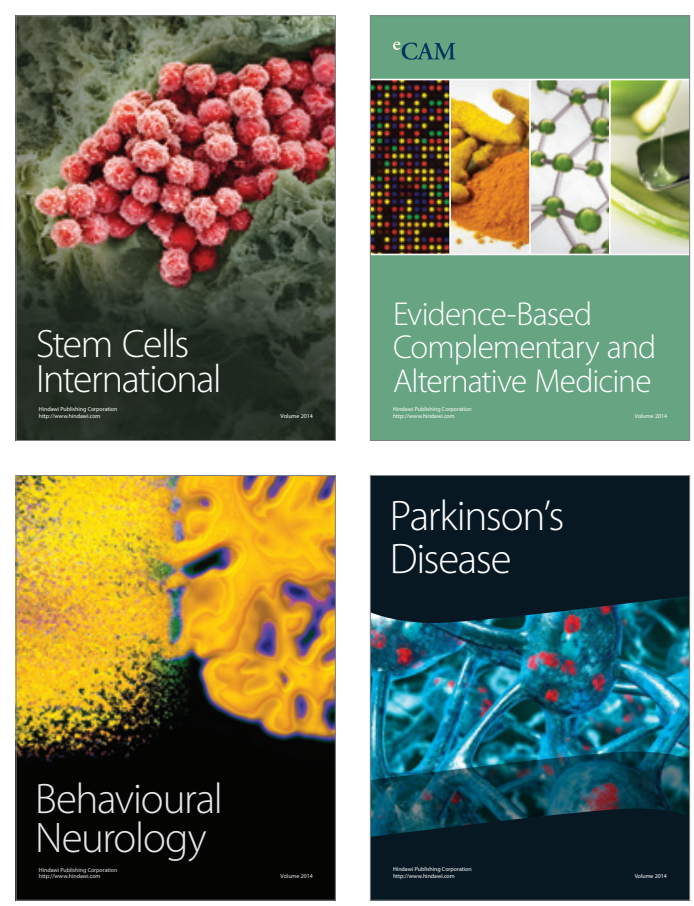

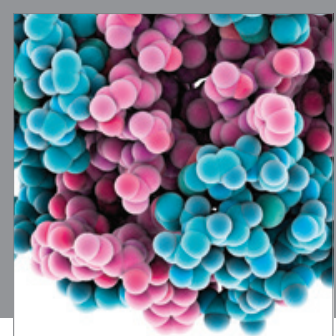

Journal of
Diabetes Research

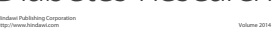

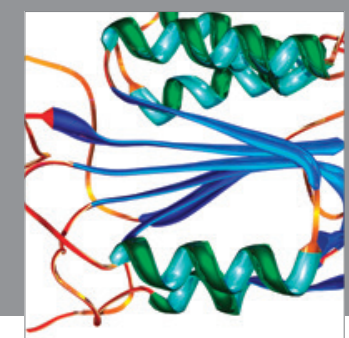

Disease Markers
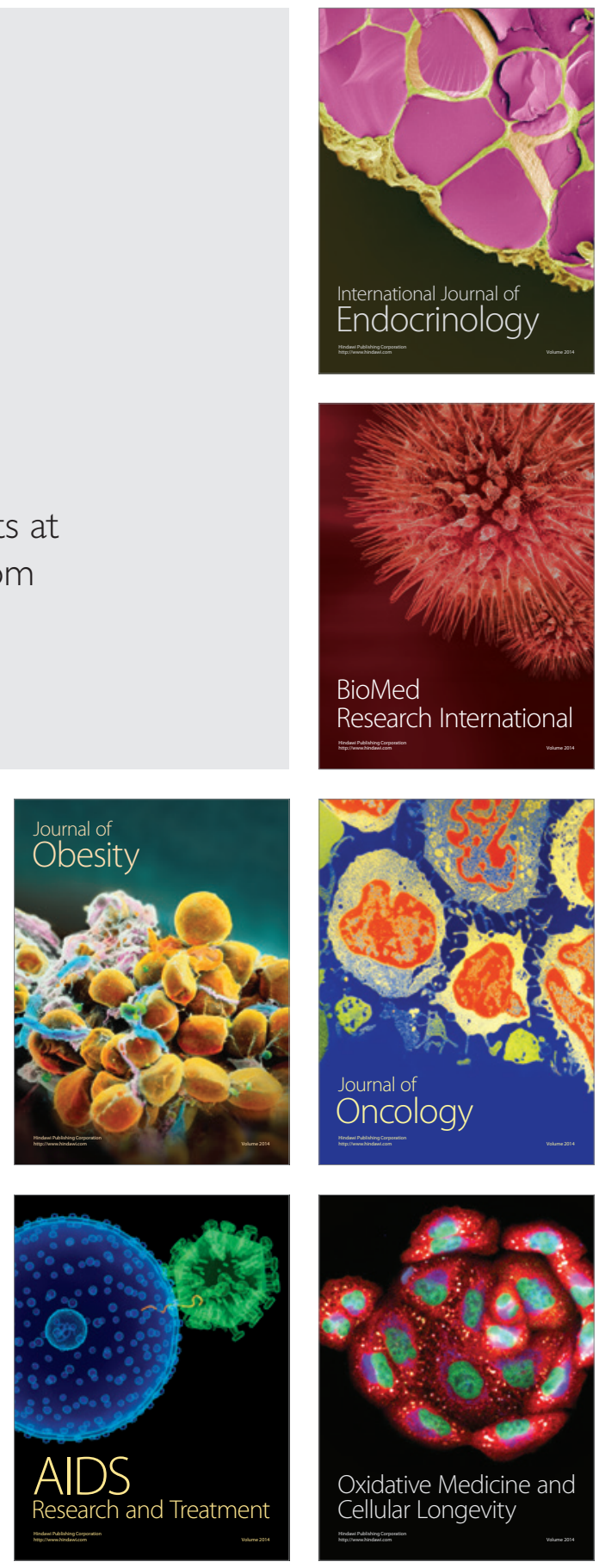